\title{
CENTROS DE DOCUMENTACION Y BIBLIOTECAS ESPECIALIZADAS EN ESPAÑA
}

\author{
Manuela Vázquez*; Alfonsa Blanco*
}

\begin{abstract}
Resumen: Con objeto de obtener una panorámica detallada de la situación de España en cuanto a recursos de información, se analiza una serie de datos contenidos en el "Directorio de Centros de Documentación y Bibliotecas Especializadas" publicado por la Dirección General de Investigación Cientifica y Técnica del Ministerio de Educación y Ciencia. Se determina la distribución geogrtfica de los Centros en las Comunidades Autónomas del Estado, tanto en valores absolutos como en relación al número de habitantes, us antiglledad, dependencia institucional, especialisación temśtica, nivel de accesibilidad y recursos humanos. Con relación a los recurso bibliograficos se clasifican los centros teniendo en cuenta el volumen de libros, revistas, revistas de resumenes y otras obras de referencia de que disponen. Por último se hace una clasificación de los centros con respecto a varios de los servicios que prestan, indicativos de su disposición hacia un mejor aprovechamiento de los recurvos bibliograficos del país.

Palabre clave: Centros de información, analisio descriptivo, Espana.
\end{abstract}

\begin{abstract}
Abatract: In order to obtain a general view of information resources in Spain, some series of data included in the "Directory of Documentation Centers and Specialised Libraries" are analysed. Geographic distribution of athor in the different Autonomous Regions related to the number of inhabitants, years of service, institutional dependence, subject specialisation, accesibility and human recources, are determined. Centers are classified according to their bibliographic resources: number of books, periodicals, abstracts journals and other reference material, as well as the services they offer.

Keywords: Information centers, descriptive analysis, Spain.
\end{abstract}

\section{Introducción}

La necesidad de conocer los centros de documentación y bibliotecas especializadas existentes en España era patente desde hace muchos años. Esta laguna del conocimiento se ha visto cubierta con la publicación del Directorio de Centros de Documentación y Bibliotecas Especializadas (1) que a finales de 1987 publicó la Dirección General de Investigación Cientifica y Técnica del Ministerio de Educación y Ciencia. Como era previsible, ha resultado ser una herramienta fundamental en el campo de la Información Cientifica, que se ha hecho imprescindible para la difusión de cualquier noticia del sector y que está siendo ventajosamente utilizada por los editores, tanto españoles como extranjeros, para esta finalidad. También los científicos y, en general, todos los usuarios de la Información Especializada disponen, gracias a dicho directorio, de una obra de referencia de gran utilidad para saber a dónde dirigirse a fin de localizar información sobre un tema concreto, conseguir una copia de un documento determinado, etc.

Aunque en el citado Directorio aparecen bastantes datos sobre los servicios, recursos y materias de especialización relativos a cada centro, éstos de una forma global, sólo aparecen ordenados según las autonomías en que se encuentran, los organismos de que dependen y las materias en las que están especializados.

* Instituto de Información y Documentación en Ciencia y Tecnología (ICYT), Madrid. CSIC.

Recibido 13-10-89 
Ejemplo de registro del Directorio

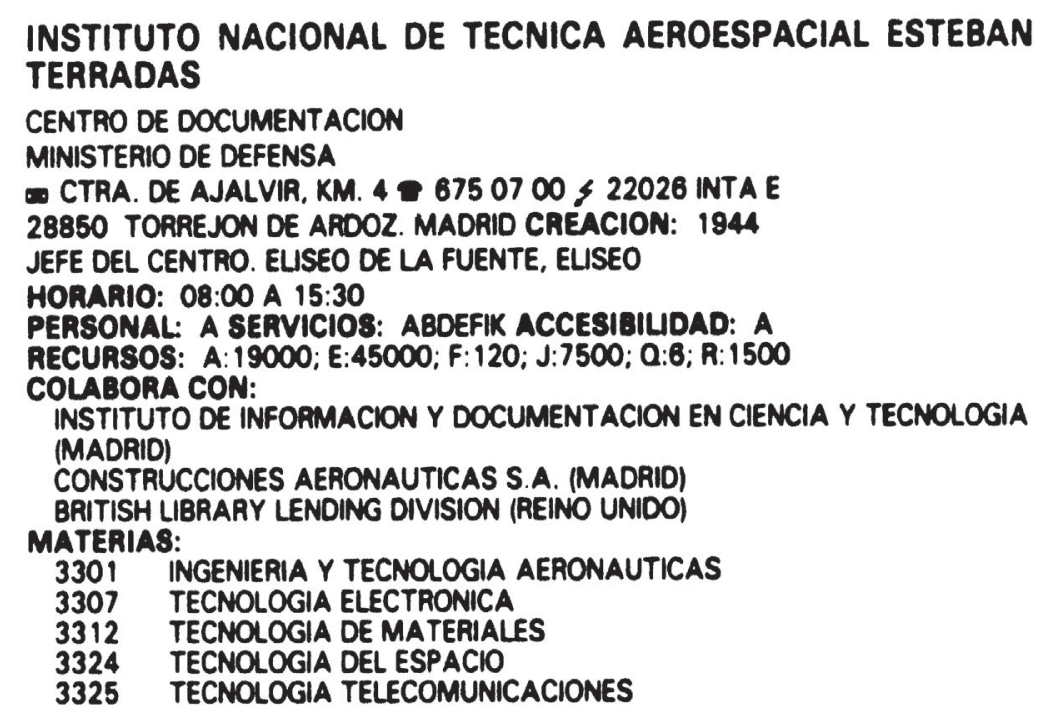

Este ejemplo ilustra la gran cantidad de datos que se suministra para cada centro. Para su interpretación, el directorio incluye una tabla de equivalentes de los códigos que integran cada registro (Tabla 1).

Este estudio pretende analizar los numerosos datos, a fin de obtener una panorámica más detallada de la situación de España en cuanto a recursos de información.

\section{Distribución Geográfica}

La figura 1 muestra la distribución de centros por autonomías, observándose que el $27 \%$ se encuentra localizado en Madrid, y más de un $17 \%$ en Cataluña, mientras que hay nueve autonomías que apenas superan entre todas el $20 \%$.

Relacionando el número de centros con el de habitantes, se obtienen los datos que figuran en la Tabla 2.

Se observa que, tanto en términos absolutos como en relación al número de habitantes, la concentración mayor de estos centros se localiza en Madrid, seguida de Cataluña. En el otro extremo se sitúa la Región Valenciana, que, aunque en términos absolutos tiene un número bastante elevado de centros, ocupa el último lugar, en unión de Castilla-La Mancha, en cuanto a su relación con el número de sus habitantes. 
Tabla 1

Interpretación de los códigos que figuran en el directorio

\begin{tabular}{|c|c|c|}
\hline REFERENCIA & CODIGO & SIGNIFICADO \\
\hline $\begin{array}{l}\text { ACCESIBILIDAD } \\
\text { (De los servicios) }\end{array}$ & $\begin{array}{l}\mathrm{A} \\
\mathrm{B} \\
\mathrm{C}\end{array}$ & $\begin{array}{l}\text { Para cualquier persona } \\
\text { Solo para personas con autorización especial } \\
\text { Particular (sólo para personas del propio Centro) }\end{array}$ \\
\hline $\begin{array}{l}\text { PEASONAL } \\
\text { (Recursos humanos) }\end{array}$ & A & $\begin{array}{l}\text { Personal con titulación universitaria, especializado } \\
\text { en Documentación o Bibliotecologia }\end{array}$ \\
\hline $\begin{array}{l}\text { RECURSOS } \\
\text { (Bibliográficos y documentales) }\end{array}$ & $\begin{array}{l}A \\
B \\
C \\
D \\
E \\
F \\
G \\
H \\
I \\
J \\
K \\
L \\
M \\
N \\
O \\
P \\
Q \\
R \\
S \\
T \\
U \\
V \\
W \\
X \\
Y \\
Z \\
\\
2 \\
3 \\
4 \\
5\end{array}$ & $\begin{array}{l}\text { Libros (monograflas) } \\
\text { Manuales técnicos } \\
\text { Tesis, Proyectos fin de Carrera, Tesinas } \\
\text { Directorios, Bibliografias, Obras de Referencia } \\
\text { Informes de Investigación. Separatas } \\
\text { Revistas técnicas y cientificas } \\
\text { Revistas de resúmenes } \\
\text { Boletines bibliogrdficos } \\
\text { Boletines, Newsletters, etc. } \\
\text { Normas } \\
\text { Patentes } \\
\text { Mapas, Planos y Fotograflas aéreas } \\
\text { Actas de Congresos } \\
\text { Pellculas, videos } \\
\text { Diapositivas } \\
\text { Discos y Cassettes } \\
\text { Microfilm } \\
\text { Microfichas } \\
\text { Folletos } \\
\text { Originales de publicidad gróficas } \\
\text { Memorias de empresas } \\
\text { Recortes de prensa } \\
\text { Unidades archivadoras } \\
\text { Grabados y dibujos } \\
\text { Pruebas psicotecnicas (tests) } \\
\text { Diarios y revistas de información general } \\
\\
\text { Piezas musicales (partituras) } \\
\text { Programas de ordenador } \\
\text { Protocolos notariales } \\
\text { Documentos de organismos internacionales } \\
\text { Registros lexicologicos }\end{array}$ \\
\hline $\begin{array}{l}\text { SERVICIOS } \\
\text { (Que proporcione el Centro) }\end{array}$ & $\begin{array}{l}A \\
B \\
C \\
D \\
E \\
F \\
G \\
H \\
I \\
J \\
K \\
L \\
M \\
N\end{array}$ & $\begin{array}{l}\text { Sala de lectura } \\
\text { Prbatemo personal } \\
\text { Prbatamo interbibliotecario } \\
\text { Reprografia de fondos propios } \\
\text { Microrreprografla } \\
\text { Reprografia, otros fondos nacionales o extranjeros } \\
\text { Servicio de Información y Referencia } \\
\text { Búsquedas retrospectivas manuales } \\
\text { Búsquedas retrospectivas mecanizadas } \\
\text { D.S.I. manual } \\
\text { D.S.I. mecanizada } \\
\text { Preparación de informes bibliográficos } \\
\text { Traducciones } \\
\text { Cursos o seminarios }\end{array}$ \\
\hline
\end{tabular}


Figura 1

Distribución del número de centros en las Comunidades Autónomas del Estado Espanol

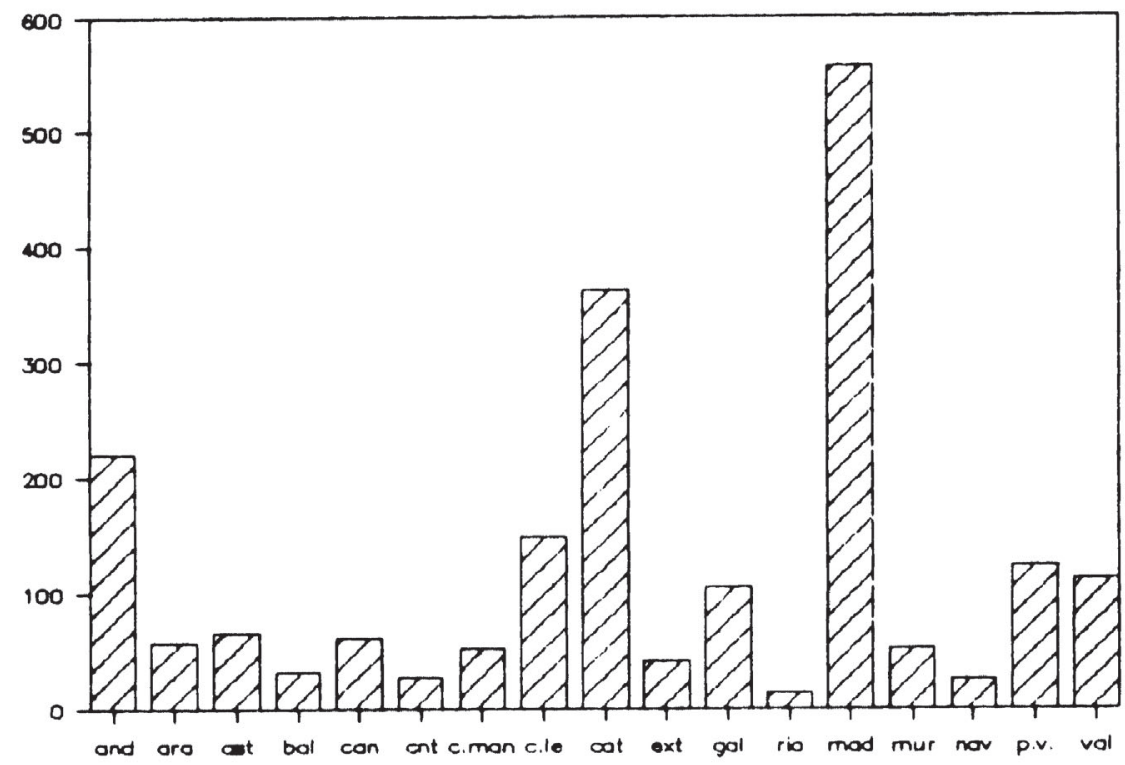

Tabla 2

Relación entre el número de centros y el de habitantes

Autonomia

Andalucia

Aragón

Asturias

Baleares

Canarias

Cantabria

Castilla-La Mancha

Castilla-León

Cataluna

Extremadura

Galicia

La Rioja

Madrid

Murcia

Navarra

Pals Vasco

Valencia

Total

\begin{tabular}{r} 
N. Cent \\
\hline 220 \\
58 \\
66 \\
32 \\
61 \\
28 \\
52 \\
148 \\
362 \\
41 \\
105 \\
14 \\
557 \\
52 \\
26 \\
123 \\
112 \\
\\
2.057
\end{tabular}

$R^{*}: N^{2}$ de centros por cada 100.000 habitantes.

\section{N2 Habitantes}

$\underline{\mathbf{R}^{*}}$

6.441 .735

1.213 .099

1.129 .572

685.088

1.444 .626

510.816

1.648 .653

2.583.159

5.958 .208

1.064 .976

2.811 .942

254.352

4.726 .986

957.903

509.002

2.141 .969

3.646 .765

37.728.831

3,4

4,8

5,8

4,7

4,2

5,6

3,1

5,7

6,1

3,8

3,7

5,5

11,8

5,4

5,1

5,7

$\mathbf{3}, \mathbf{1}$

5,5 


\section{Distribución por funciones}

La Tabla 3 contiene clasificados, también por autonomias, los diferentes centros agrupados por su función en: bibliotecas, unidades de información y documentación y los que realizan ambas funciones.

Tabla 3

Distribución de las bibliotecas, unidades de información y centros mixtos por autonomias

\begin{tabular}{|c|c|c|c|c|}
\hline Autonomias & ibliotecas & Cent. Doc. & Centros Mixtos & Total \\
\hline Andalucía & 195 & 17 & 8 & 220 \\
\hline Aragón & 54 & 3 & 1 & 58 \\
\hline Asturias & 57 & 8 & 1 & 66 \\
\hline Baleares & 26 & 4 & 2 & 32 \\
\hline Canarias & 55 & 5 & 1 & 61 \\
\hline Cantabria & 25 & 1 & 2 & 28 \\
\hline Castilla-La Mancha & 50 & 1 & 1 & 52 \\
\hline Castilla-León & 138 & 8 & 2 & 148 \\
\hline Cataluña & 294 & 49 & 19 & 362 \\
\hline Extremadura & 40 & 1 & - & 41 \\
\hline Galicia & 95 & 8 & 2 & 105 \\
\hline La Rioja & 11 & 3 & - & 14 \\
\hline Madrid & 389 & 119 & 49 & 557 \\
\hline Murcia & 45 & 6 & 1 & 52 \\
\hline Navarra & 21 & 5 & - & 26 \\
\hline País Vasco & 97 & 21 & 5 & 123 \\
\hline Valencia & 95 & 14 & 3 & 112 \\
\hline Total & 1.687 & 273 & 97 & 2.057 \\
\hline
\end{tabular}

Llama la atención el pequeño número de centros de documentación existentes $y$, sobre todo, su desigual reparto en las diferentes autonomias. A este respecto las que tienen un menor número de centros de documentación son: Extremadura, Castilla-La Mancha, Cantabria y La Rioja.

\section{Antigüedad}

La tabla 4 muestra la distribución de los centros y bibliotecas españolas en función de su antigüedad. Se observa que más del $50 \%$ tienen una antigüedad comprendida entre 10 y 50 años y que 432 centros (21\%) fueron creados en los últimos 10 años. Entre los más antiguos se encuentran 25 centros cuya creación tuvo lugar hace más de 200 años. Esos centros se recogen ordenados, en función de su antigüedad, en la tabla 5 , en la que para cada centro se indica la autonomia en que se encuentra, año de creación y organismo a que se encuentra adscrito actualmente. Puede verse que 9 de los centros (36\%), con más de 200 años de antigüedad, pertenecen a la Universidad; 5 de ellos (20\%) son de diferentes Asociaciones Profesionales o Culturales, y que a diversas Instituciones Religiosas y a Reales Academias se adscriben por igual otros 8 centros. En el grupo se encuentra también un centro de dependencia ministerial, la Biblioteca del Ministerio de Asuntos Exteriores. Por último, los dos centros restantes pertenecen actualmente al CSIC, aunque, dado que este organismo fue creado en 1949, es evidente que ambos tenian ya una larga 
vida cuando fueron adscritos a dicho organismo. Se trata de la Biblioteca del Real Jardin Botánico, fundada en 1755, y la Biblioteca de Zoologia del Museo de Ciencias Naturales, que data de 1766.

Tabla 4

Distribución de los centros por autonomias

$y$ aflos de antiguledad

\begin{tabular}{|c|c|c|c|c|c|c|c|}
\hline \multirow[t]{2}{*}{ Autonomia } & & \multicolumn{6}{|c|}{ Antiguledad en anos } \\
\hline & $\leq 10$ & $10-50$ & $\underline{51-100}$ & $101-200$ & $\geq 200$ & S. D.* & Total \\
\hline Andalucia & 43 & 116 & 17 & 10 & 2 & 32 & 220 \\
\hline Aragón & 13 & 25 & 5 & 4 & 1 & 10 & 58 \\
\hline Asturias & 16 & 33 & 1 & 1 & 3 & 12 & 66 \\
\hline Baleares & 9 & 10 & 5 & 3 & - & 5 & 32 \\
\hline Canarias & 12 & 31 & 1 & 1 & 1 & 15 & 61 \\
\hline Cantabria & 4 & 12 & 6 & - & - & 6 & 28 \\
\hline Castilla-La Mancha & 9 & 31 & 5 & 1 & 1 & 5 & 52 \\
\hline Castilla-León & 42 & 64 & 15 & 1 & - & 26 & 148 \\
\hline Cataluña & 67 & 196 & 36 & 19 & 3 & 11 & 362 \\
\hline Extremadura & 8 & 25 & 3 & - & - & 5 & 41 \\
\hline Galicia & 16 & 48 & 8 & 4 & 4 & 25 & 105 \\
\hline La Rioja & 4 & 6 & - & 1 & - & 3 & 14 \\
\hline Madrid & 96 & 306 & 39 & 20 & 6 & 90 & 557 \\
\hline Murcia & 17 & 24 & 4 & 1 & - & 6 & 52 \\
\hline Navarra & 6 & 12 & 1 & 1 & - & 6 & 26 \\
\hline Paía Vasco & 39 & 56 & 5 & 2 & 1 & 20 & 123 \\
\hline Valencia & 31 & 49 & 8 & 3 & 3 & 18 & 112 \\
\hline Total & 432 & 1044 & 159 & 72 & 25 & 325 & 2057 \\
\hline
\end{tabular}

* Sin datos. Este dato no figura en el directorio

\section{Dependencia institucional}

La tabla 6 recoge la adscripción institucional de los centros y bibliotecas especializados españoles.

Se observa que el mayor porcentaje de estos centros pertenece a la Universidad, seguida de los que pertenecen a Organismos Públicos de Investigación (OPIS). En este grupo se incluyen los centros de documentación y bibliotecas especializadas del CSIC, que es el OPI que posee un número más elevado de centros de este tipo. Es también elevado el número de centros pertenecientes a Asociaciones Cientificas, Culturales y Profesionales. 
Relación de las bibliotecas creadas hace más de 200 años

Nombre

1. Bibl.Abadia de Montserrat

2. Bibl.Prov. Franciscana de Santiago

3. Bibl.Gen. Univ. Sevilla

4. Bibl.Fac. Derecho de Santiago

5. Bibl.del Santuario de $N^{2}$ Sra. de Aránzazu

6. Bibl.Gen. Universidad Santiago

7. Bibl.Central Universidad Oviedo

8. Bibl.Real Academia Lengua

9. Bibl.Gen. Universidad Granada

10.Bibl.Gen. Universidad Zaragoza

11. Bibl.Fac. Derecho Universidad Oviedo

12. Bibl.Minist. Asuntos Exteriores

13. Bibl.Fac. Bellas Artes Univ. Complutense

14. Bibl.Real Jardín Botánico

15. Bibl.Colegio Abogados Valencia

16. Bibl.Real Acad. Medicina y Cirugía de Galicia

17. Bibl.Real Acad. Bellas Artes de San Carlos

18. Bibl.Real Acad. Medicina Barcelona

19. Bibl.Públ. Episcopal Semin. Conciliar Barcelona

20. Bibl.Colegio Abogados Oviedo

21. Bibl.Real Soc. Económica Matritense

Amigos del País

22. Bibl.Zoología Museo Ciencias Naturales

23. Bibl.Soc. Econom. Amigos del Pals Valenciano

24.Bibl.Real Soc. Econom. Amigos Pais Tenerife

25. Bibl.Esc. Universidad Politécnica Almadén
Año de

Autonomia creación Organismo

$\begin{array}{ll}\text { Cataluña } & 1.029 \\ \text { Galicia } & 1.240 \\ \text { Andalucia } & 1.501 \\ \text { Galicia } & 1.504 \\ \text { País Vasco } & 1.512 \\ \text { Galicia } & 1.550 \\ \text { Asturias } & 1.608 \\ \text { Madrid } & 1.713 \\ \text { Andalucia } & 1.740 \\ \text { Aragón } & 1.742 \\ \text { Asturias } & 1.750 \\ \text { Madrid } & 1.750 \\ \text { Madrid } & 1.752 \\ \text { Madrid } & 1.755 \\ \text { Valencia } & 1.759 \\ \text { Galicia } & 1.765 \\ \text { Valencia } & 1.768 \\ \text { Cataluña } & 1.770 \\ \text { Catalufá } & 1.772 \\ \text { Asturias } & 1.775 \\ & \\ \text { Madrid } & 1.775 \\ \text { Madrid } & 1.776 \\ \text { Valencia } & 1.776 \\ \text { Canarias } & 1.777 \\ \text { Cast-Mancha } & 1.777\end{array}$

Inst. Relig.

Inst. Relig.

Universidad

Universidad

Inst. Relig.

Universidad

Universidad

Real. Acad.

Universidad

Universidad

Universidad

Ministerio

Universidad

CSIC

Asoc. Prof.

Real Acad.

Real Acad.

Real Acad.

Inst. Relig.

Asoc. Prof.

Asoc. Prof.

CSIC

Asoc. Prof.

Asoc. Prof.

Universidad

\section{Especialización temática}

Examinando la distribución de los centros en relación con su especialización temática, se encuentran los resultados de la Tabla 7. Las materias consideradas van acompañadas de los códigos correspondientes a la disciplina según la clasificación de la "Nomenclatura Internacional de la UNESCO para los campos de Ciencia y Tecnología" (2) que es utilizada en el Directorio. La mayor parte de los centros contempla varias disciplinas, con una media de casi cuatro disciplinas por centro. Las materias atendidas por un número mayor de centros son, en orden decreciente, Medicina, Ciencias Tecnológicas, Historia, Economía, Agronomía y Matemáticas. Por el contrario, entre las más desatendidas se encuentran Lógica, Etica, Astronomía y Astrofísica y Demografía. En lineas generales, tanto las disciplinas de ciencias como las de $\mathrm{Hu}-$ manidades (incluyendo aqui las Ciencias Sociales) son cubiertas por un número parecido de centros. 
Adscripción institucional de los centros y bibliotecas

\begin{tabular}{|c|c|c|}
\hline & $\underline{N^{2}}$ & \$ \\
\hline $\begin{array}{l}\text { Organismos Ministeriales Administrativos } \\
\text { Organismos Autonómicos }\end{array}$ & 117 & $\begin{array}{r}5,7 \\
127\end{array}$ \\
\hline Cortes Generales y Organismos Constitucionales & 4 & $\begin{array}{r}10,1 \\
0,2\end{array}$ \\
\hline Instituciones Judiciales & 25 & 1,2 \\
\hline Instituciones Polfticas & 6 & 0,3 \\
\hline Administraciones locales & & \\
\hline Ayuntamientos & 47 & 2,3 \\
\hline Diputaciones & 58 & 2,8 \\
\hline Universidades & 643 & 31,2 \\
\hline Centros de Ensefiansa Integrada & 26 & 1,3 \\
\hline OPIS (*) & 351 & 17,1 \\
\hline Asoc. Cientificas, Culturales o Profesionales & 178 & 8,6 \\
\hline Fundaciones y Patronatos & 57 & 2,8 \\
\hline Camaras Oficiales de Comercio e Industria & 39 & 1,9 \\
\hline Entidades Financieras & 26 & 1,3 \\
\hline Entidades Privadas & 147 & 7,1 \\
\hline Instituciones Religiosas & 47 & 2,3 \\
\hline Organismos Extranjeros o Internacionales & & \\
\hline radicados en Espana & 24 & 1,2 \\
\hline Total & 2.057 & 100 \\
\hline
\end{tabular}

* En este grupo eatá incluido el CSIC, con 109 centros, lo que supone ma del 5\% del total Tabla 7

Disciplinas tematicas y número de centros que las atienden

\begin{tabular}{|c|c|c|}
\hline Clasif. Unesco & Materia & centros \\
\hline 11 & Logrica & 16 \\
\hline 12 & Matematicas & 286 \\
\hline 21 & Astronomia y Astrofisica & 38 \\
\hline 22 & Física & 251 \\
\hline 23 & Química & 331 \\
\hline 24 & Ciencias de la Vida & 350 \\
\hline 25 & Ciencias de la Tierra y el Espacio & 270 \\
\hline 31 & Ciencias Agronómicas & $\mathbf{3 2 0}$ \\
\hline 32 & Ciencias Médicas & 966 \\
\hline 33 & Ciencias Tecnologricas & 962 \\
\hline 51 & Antropología & 144 \\
\hline 52 & Demografia & 42 \\
\hline 53 & Ciencias Económicas & 654 \\
\hline 54 & Geografia & 145 \\
\hline 55 & Historia & 923 \\
\hline 56 & Ciencias Juridicas. Leyes & 520 \\
\hline 67 & Lingurstica & 173 \\
\hline 58 & Pedagoria & 233 \\
\hline 59 & Ciencias Polfticas & 194 \\
\hline 61 & Paicología & 270 \\
\hline 62 & Bellas Artes. Letras & 469 \\
\hline 63 & Sociología & 256 \\
\hline 71 & Etica & 23 \\
\hline 72 & Filosofla & 122 \\
\hline
\end{tabular}


7. Grado de accesibilidad

El directorio considera tres niveles diferentes de accesibilidad de los centros:
A. para cualquier persona
B. sólo para personas con autorización especial
C. privado (sólo para personas del propio centro).

La tabla 8 recoge la distribución numérica de los centros existentes en cada autonomia según los niveles indicados, y los porcentajes respectivos. Se observa que sólo algo más del $40 \%$ de los centros son de acceso libre para cualquier persona y que en 10 comunidades autónomas se supera este porcentaje. Los centros accesibles sólo al personal que trabaja en ellos constituyen un elevado porcentaje del total $(23,4 \%)$; la mayoría de ellos son centros de documentación de empresas privadas, por lo que suele haber un número mayor en las regiones con más industrias. Una excepción a este respecto la constituye la comunidad de Castilla-La Mancha, en la que, de los 19 centros de accesibilidad $\mathrm{C}$, sólo tres son de empresas industriales.

Tabla 8

Clasificación de los centros por nivel de accesibilidad

\begin{tabular}{|c|c|c|c|c|c|c|c|}
\hline \multirow[t]{2}{*}{ Autonomía } & \multicolumn{7}{|c|}{ Nivel de accesibilidad } \\
\hline & $\mathbf{A}$ & $\%$ & $\mathbf{B}$ & $\%$ & $\mathrm{C}$ & $\%$ & $\mathrm{~T}$ \\
\hline Andalucía & 95 & 43,2 & 79 & 35,9 & 46 & 20,9 & 220 \\
\hline Aragón & 29 & 50 & 14 & 24,1 & 15 & 25,9 & 58 \\
\hline Asturias & 23 & 34,8 & 23 & 34,8 & 20 & 30,3 & 66 \\
\hline Baleares & 14 & 43,8 & 13 & 40,6 & 5 & 15,6 & 32 \\
\hline Canarias & 31 & 50,8 & 16 & 26,2 & 14 & 23 & 61 \\
\hline Cantabria & 14 & 50 & 9 & 32,1 & 5 & 17,9 & 28 \\
\hline Castilla-La Mancha & 12 & 23,1 & 21 & 40,4 & 19 & 36,5 & 52 \\
\hline Castilla-León & 58 & 39,2 & 64 & 43,2 & 26 & 17,6 & 148 \\
\hline Cataluña & 176 & 48,6 & 100 & 27,6 & 86 & 23,8 & 362 \\
\hline Extremadura & 16 & 39 & 15 & 36,6 & 10 & 24,4 & 41 \\
\hline Galicia & 41 & 39 & 42 & 40 & 22 & 21 & 105 \\
\hline La Rioja & 9 & 64,3 & 4 & 28,6 & 1 & 7,1 & 14 \\
\hline Madrid & 207 & 37,2 & 213 & 38,2 & 137 & 24,6 & 557 \\
\hline Murcia & 21 & 40,4 & 16 & 30,8 & 15 & 28,8 & 52 \\
\hline Navarra & 13 & 50 & 9 & 34,6 & 4 & 15,4 & 26 \\
\hline País Vasco & 53 & 43 & 43 & 35 & 27 & 22 & 123 \\
\hline Valencia & 58 & 51,8 & 25 & 22,3 & 29 & 25,9 & 112 \\
\hline Total & 870 & 42,3 & 706 & 34,3 & 481 & 23,4 & 2057 \\
\hline
\end{tabular}

\section{Recursos humanos}

En relación con su dotación en recursos humanos, el directorio da pocos datos, ya que sólo consigna el hecho de si el centro o biblioteca dispone de personal de titulación universitaria superior o no, sin indicar tampoco su nú- 
mero, y, evidentemente, existe una gradación, a este respecto, mayor que la que representa este único dato, aunque sea importante y significativo.

La tabla 9 muestra el número de centros de documentación y bibliotecas en que existe personal con titulación universitaria en las 17 autonomías del estado español. En ella figura el número total de centros que cuenta con personal de titulación universitaria y el porcentaje que éstos suponen del total.

Se observa que Madrid y Cataluña tienen aproximadamente el $50 \%$ de sus centros dotados con personal universitario. La media nacional se aproxima al $37 \%$, pero sólo dos comunidades tienen valores cercanos a la media, estando las restantes bastante por debajo de este valor, con Castilla-La Mancha, Murcia y Baleares en los últimos lugares.

Tabla 9

$N^{2}$ de centros con personal universitario

\begin{tabular}{|c|c|c|c|}
\hline Autonomía & $\begin{array}{l}\mathrm{N}^{2} \text { total de } \\
\text { centros }\end{array}$ & $\begin{array}{l}\mathrm{N}^{2} \text { centros con } \\
\text { personal universit. }\end{array}$ & $\%$ \\
\hline Andalucía & 220 & 54 & 24,5 \\
\hline Aragón & 58 & 21 & 36,2 \\
\hline Asturias & 66 & 18 & 27,2 \\
\hline Baleares & 32 & 5 & 15,6 \\
\hline Canarias & 61 & 17 & 27,8 \\
\hline Cantabria & 28 & 6 & 21,4 \\
\hline Castilla-La Mancha & 52 & 7 & 13,5 \\
\hline Castilla-León & 148 & 40 & 27 \\
\hline Cataluña & 362 & 177 & 48,9 \\
\hline Extremadura & 41 & 9 & 22 \\
\hline Galicia & 105 & 25 & 23,8 \\
\hline La Rioja & 14 & 5 & 35,7 \\
\hline Madrid & 557 & 288 & 51,7 \\
\hline Murcia & 52 & 8 & 15,4 \\
\hline Navarra & 26 & 7 & 27 \\
\hline País Vasco & 123 & 38 & 31 \\
\hline Valencia & 112 & 34 & 30,3 \\
\hline Total & 2.057 & 759 & 36,9 \\
\hline
\end{tabular}

Porcentaje medio $=36,9 \%$

\section{Recursos bibliográficos}

El directorio analizado recoge un gran número de datos relativos a los recursos bibliográficos con que cuentan los centros que lo integran (a diferencia de lo que ocurre con los datos de personal). Sin embargo no todos los tipos de recursos en él reseñados son de igual importancia para todos los centros, ya que en algunos casos su interés depende de la temática de que se ocupa el centro; éste, por ejemplo, es el caso de las patentes o las partituras musicales. Es difícil que puedan ser aportados otros datos por centros que no estén automatizados (por ejemplo, número de folletos o de recortes de prensa). Por otra parte, incluso la cantidad de libros y revistas, aun siendo importante, tendria que ser considerada en relación con la amplitud o diversificación de la cobertura temática de los centros, y teniendo presente la tradición o modernidad de la disciplina de que tratan. 
Sin embargo, reconociendo que una clasificación relativa de las bibliotecas y centros de documentación con respecto a sus recursos bibliográficos debería tener presente éstos y otros factores, dentro del presente análisis se ha considerado la clasificación de los centros de manera absoluta con respecto a unos recursos bibliográficos que tienen la mayoría de ellos, como son los libros y las revistas técnicas y científicas. Se han clasificado también con respecto a otros dos tipos de recursos: revistas de resúmenes y obras de referencia, por su gran importancia para facilitar el acceso a la información.

Las tablas 10 a 13 contienen estos datos, agrupados en bloques, para facilitar su tabulación.

Con respecto al número de libros se observa que el mayor porcentaje de centros $(48,6 \%)$ tienen entre 1000 y 10.000 libros; hay un $17 \%$ que tienen entre 10.000 y 50.000, y sólo 38 centros tienen más de 100.000 libros.

En relación a las revistas científicas y técnicas, un $56,4 \%$ tiene menos de 100 títulos, y sólo 36 centros poseen más de 1000 títulos. En el directorio figura el Instituto Nacional de Investigaciones Agrarias con 15.000 títulos de revistas. Dada la importancia y excepcionalidad de la cifra, se decidió confirmarla, encontrándose que se trataba de un error de transcripción, y el correcto era 1500 .

Tabla 10

Clasificación de los centros con repecto al número de libros que poseen

\begin{tabular}{|c|c|c|c|c|c|c|c|}
\hline Autonomia & $\leq 1000$ & $\begin{array}{l}1001- \\
10000 \\
\end{array}$ & $\begin{array}{l}10001- \\
\underline{50000}\end{array}$ & $\begin{array}{l}50001- \\
100000 \\
\end{array}$ & $\geq 100000$ & $\underline{\text { S.D. }}{ }^{*}$ & Total \\
\hline Andalucia & 51 & 114 & 40 & 4 & 4 & 7 & 220 \\
\hline Aragón & 10 & 35 & 5 & 2 & & 6 & 58 \\
\hline Asturias & 19 & 33 & 7 & 2 & 1 & 4 & 66 \\
\hline Baleares & 12 & 12 & 4 & 2 & - & 2 & 32 \\
\hline Canarias & 20 & 28 & 10 & 2 & 1 & - & 61 \\
\hline Cantabria & 6 & 16 & 2 & - & 1 & 3 & 28 \\
\hline Castilla-La Mancha & 23 & 21 & 6 & - & - & 2 & 52 \\
\hline Castilla-León & 38 & 77 & 22 & 5 & 3 & 3 & 148 \\
\hline Cataluña & 82 & 179 & 69 & 10 & 9 & 13 & 362 \\
\hline Extremadura & 15 & 18 & 6 & 1 & - & 1 & 41 \\
\hline Galicia & 31 & 48 & 16 & 6 & 1 & 3 & 105 \\
\hline La Rioja & 4 & 8 & 1 & - & - & 1 & 14 \\
\hline Madrid & 114 & 257 & 121 & 27 & 11 & 27 & 557 \\
\hline Murcia & 19 & 26 & 5 & - & - & 2 & 52 \\
\hline Navarra & 2 & 19 & 2 & 1 & 1 & 1 & 26 \\
\hline Pars Vasco & 33 & 55 & 21 & 5 & 2 & 7 & 123 \\
\hline Valencia & 32 & 54 & 14 & 3 & 4 & 5 & 112 \\
\hline Total & 511 & 1000 & 351 & 70 & 38 & 87 & 2057 \\
\hline$\%$ & 24,9 & 48,6 & 17,1 & 3,4 & 1,8 & 4,2 & \\
\hline
\end{tabular}

*S.D. Sin datos. Este dato no figura en el directorio. 
Tabla 11

Clasificación de los centros en relación con el número de revistas cientificas que poseen

\begin{tabular}{|c|c|c|c|c|c|}
\hline Autonomía & $\leq 100$ & $101-1000$ & $1001-5000$ & $\underline{\text { S.D.* }}$ & Total \\
\hline Andalucia & 131 & 60 & 5 & 24 & 220 \\
\hline Aragón & 32 & 19 & - & 7 & 58 \\
\hline Anturias & 37 & 18 & - & 11 & 66 \\
\hline Baleares & 18 & 6 & - & 8 & 32 \\
\hline Canarias & 36 & 10 & 1 & 15 & 61 \\
\hline Cantabria & 13 & 9 & - & 6 & 28 \\
\hline Cantilla-La Mancha & 37 & 6 & - & 9 & 52 \\
\hline Castilla-Loón & 89 & 34 & 2 & 23 & 148 \\
\hline Cataluna & 204 & 113 & 5 & 40 & 362 \\
\hline Extremadura & 34 & 5 & - & 2 & 41 \\
\hline Galicia & 67 & 23 & - & 15 & 105 \\
\hline La Rioja & 8 & $\mathbf{s}$ & - & 3 & 14 \\
\hline Madrid & 280 & 197 & 18 & 62 & 557 \\
\hline Murcia & 32 & 8 & 1 & 11 & 52 \\
\hline Navarra & 15 & 5 & 2 & 4 & 26 \\
\hline Paí Vasco & 64 & 43 & 1 & 15 & 123 \\
\hline Valencia & 65 & 35 & 2 & 10 & 112 \\
\hline Total & 1161 & 594 & 37 & 265 & 2057 \\
\hline$\%$ & 56,4 & 28,9 & 1,8 & 12,9 & \\
\hline
\end{tabular}

*S.D. Sin datos. Este dato no figura en el directorio.

Tabla 12

Clasificación de los centros con respecto al número de revistas de resúmenes que poseen

\begin{tabular}{|c|c|c|c|c|c|c|c|}
\hline Autonomis & $\leq 10$ & $11-20$ & $\underline{21-50}$ & $\underline{51-100}$ & $\geq 100$ & $\underline{\text { S.D.* }}$ & Total \\
\hline Andalucia & 43 & 4 & 5 & 2 & - & 166 & 220 \\
\hline Aragón & 9 & 2 & - & 1 & 1 & 45 & 68 \\
\hline Asturias & 10 & - & - & - & - & 56 & 66 \\
\hline Baleares & 2 & - & - & - & - & 90 & $\mathbf{3 2}$ \\
\hline Canarias & 8 & 1 & 2 & 1 & - & 49 & 61 \\
\hline Cantabria & 4 & - & 1 & - & - & 23 & 28 \\
\hline Castilla-La Mancha & 9 & 1 & - & - & 1 & 41 & 62 \\
\hline Castilla-León & 17 & 5 & 1 & 1 & 1 & 123 & 148 \\
\hline Cataluña & 83 & 6 & 5 & 2 & 1 & 263 & 362 \\
\hline Extremadura & 9 & - & - & - & - & 32 & 41 \\
\hline Galicia & 18 & 3 & 3 & - & - & 81 & 105 \\
\hline La Rioja & 1 & - & - & - & - & 13 & 14 \\
\hline Madrid & 107 & 18 & 10 & 5 & 3 & 414 & 657 \\
\hline Murcia & 8 & - & - & - & - & 44 & 62 \\
\hline Navarra & 2 & - & 1 & - & - & 23 & 26 \\
\hline Paí Vasco & 23 & 4 & 2 & 1 & - & 98 & 123 \\
\hline Valencia & 24 & 4 & - & 3 & 2 & 79 & 112 \\
\hline Total & 377 & 48 & 30 & 16 & 9 & 1575 & 2057 \\
\hline$\%$ & 18,3 & 2,3 & 1,4 & 0,7 & 0,4 & 76,5 & \\
\hline
\end{tabular}

*S.D. Sin datos. Eate dato no figura en el directorio. 
Clasificación de los centros en relación con el número de obras de referencia de que disponen

\begin{tabular}{|c|c|c|c|c|c|c|c|}
\hline Autonomía & $\leq 100$ & $\underline{101-200}$ & $\underline{201-500}$ & $\underline{501-1000}$ & $\geq 1000$ & $\underline{\text { S.D. } *}$ & Total \\
\hline Andalucía & 35 & 5 & 6 & 2 & 4 & 168 & 200 \\
\hline Aragón & 12 & 1 & 3 & - & 1 & 41 & 58 \\
\hline Asturias & 7 & 1 & - & - & 1 & 57 & 66 \\
\hline Baleares & - & - & - & 1 & 1 & 30 & 32 \\
\hline Canarias & 9 & 1 & 3 & 1 & & 47 & 61 \\
\hline Cantabria & 2 & - & 1 & - & - & 25 & 28 \\
\hline Castilla-La Mancha & 8 & 1 & 1 & - & 1 & 41 & 52 \\
\hline Castilla-León & 20 & 5 & 3 & 2 & 2 & 116 & 148 \\
\hline Cataluña & 71 & 9 & 15 & 2 & 11 & 254 & 362 \\
\hline Extremadura & 9 & - & - & - & 1 & 31 & 41 \\
\hline Galicia & 15 & 2 & 2 & 1 & 1 & 84 & 105 \\
\hline La Rioja & 3 & 1 & 1 & - & - & 9 & 14 \\
\hline Madrid & 111 & 24 & 27 & 10 & 15 & 370 & 557 \\
\hline Murcia & 11 & 2 & - & 1 & - & 38 & 52 \\
\hline Navarra & 6 & - & 1 & 1 & - & 18 & 26 \\
\hline País Vasco & 26 & 2 & 4 & 2 & 1 & 88 & 123 \\
\hline Valencia & 31 & 1 & 4 & - & 2 & 74 & 112 \\
\hline Total & 376 & 55 & 71 & 23 & 41 & 1490 & 2057 \\
\hline$\%$ & 18,27 & 2,6 & 3,4 & 1,1 & 2,0 & 72,4 & \\
\hline
\end{tabular}

*S.D. Sin datos. Este dato no figura en el directorio.

En cuanto a las revistas de resúmenes, hay que indicar que 1575 centros (más del $75 \%$ ) no poseen en su fondos este tipo de fuentes secundarias de información, o al menos no las contabilizan separadamente; que el $18 \%$ tienen menos de 10 títulos, y sólo $9(0,4 \%)$ tienen más de 100 titulos. Se pone de manifiesto la escasez de este tipo de recursos a nivel de todo el país y en particular en las comunidades de Baleares, La Rioja y Navarra.

Al considerar las obras de referencia, se observan resultados semejantes a los que se contemplan con respecto a las revistas de resúmenes. También en este caso un $72 \%$ de los centros no aportan datos sobre ello y el $18 \%$ posee menos de 100 obras de este tipo. Destaca asímismo la escasa disponibilidad de este tipo de obras en España, y en especial en las Comunidades Autónomas de Baleares, Cantabria y La Rioja.

\section{Servicios}

En este apartado se clasifican los centros con respecto a varios de los servicios que figuran en el directorio analizado:

C. Préstamo interbibliotecario

D. Reprografía de fondos propios

F. Reprografia procedente de otros fondos

H. Búsquedas retrospectivas manuales

I. Búsquedas retrospectivas automatizadas

N. Cursos o seminarios que imparte el centro 
La letra que aparece delante de cada tipo de servicio es la clave que tiene asignada en el directorio (ver Tabla 1).

Se han considerado estos servicios por estimarse que son los más indicativos de la disposición de cada centro hacia un máximo aprovechamiento de los recursos de información, tanto propios como de otras bibliotecas o servicios de información, para facilitar la difusión del conocimiento.

Mención aparte merece la actividad de impartir cursos o seminarios, incluida en el directorio entre los servicios. Aunque hay múltiples razones para considerarla separadamente, se ha incluido en este apartado con objeto de mantener los resultados de este análisis en el mismo orden en que figuran los datos en el directorio analizado.

La tabla 14 recoge los resultados del número de centros que realizan los servicios citados en las diecisiete comunidades autónomas de España. En cada columna se indica el número absoluto y el porcentaje que ese número supone respecto al total de centros de cada comunidad. Se da también el número total y el porcentaje para todo el pais.

En relación al préstamo interbibliotecario, lo realizan 449 centros de todo el pais, lo que supone un porcentaje del $21,8 \%$ de los centros. Este pobre resultado es mucho más bajo en las comunidades autónomas de Baleares, Canarias y Extremadura, en donde no llega al 10\% los centros que lo tienen establecido. En total once comunidades tienen un valor inferior a la ya insuficiente media nacional (3).

Menos explicable aún es la disposición de los centros hacia la reprografía de fondos propios, en la que la media del pais no alcanza el $60 \%$. Ocupa el último lugar al respecto Murcia, donde sólo un $28,8 \%$ de los centros realizan este elemental servicio.

En lo que concierne a la reprografia de otros fondos nacionales o extranjeros, los porcentajes descienden marcadamente, y sólo la realizan 265 centros $(12,9 \%)$. Son las comunidades autónomas de Aragón, Baleares y Extremadura las que están peor atendidas en este aspecto. Hay que indicar aquí que 72 de esos 265 centros señalan al ICYT o al ISOC como primer centro con el que mantienen relación, lo que podria indicar que realizan a través de dichos centros la reprografia de otros fondos.

El servicio de búsquedas retrospectivas manuales es prestado por 485 centros $(23,6 \%)$, cifra que coincide prácticamente con el número de centros que dan datos sobre el número de revistas de resúmenes que tienen (ver Tabla 12). De nuevo son Baleares y Castilla-La Mancha las comunidades que cuentan con menos centros que realicen este servicio.

Las búsquedas retrospectivas automatizadas sólo son realizadas por un $7,4 \%$ de los centros, y en las comunidades de Castilla-La Mancha, Galicia y La Rioja no figura en el directorio ningún centro que las realice.

Por último, el porcentaje de centros que realiza la actividad de impartir cursos o seminarios es de un $16,5 \%$, y en todas las comunidades autónomas hay algún centro que la lleva a cabo. 
Tabla 14

Clasificación de los centros con relación

a los servicios que imparten

\begin{tabular}{|c|c|c|c|c|c|c|c|c|c|c|c|c|}
\hline \multirow[b]{2}{*}{ Autonomia } & \multicolumn{2}{|r|}{ C } & \multicolumn{2}{|r|}{$D$} & \multicolumn{2}{|r|}{$\mathbf{F}$} & \multicolumn{2}{|r|}{$\mathbf{H}$} & \multicolumn{2}{|r|}{ I } & \multirow[b]{2}{*}{ N2 } & \multirow{2}{*}{$\underline{L}$} \\
\hline & N2 & \% & $\underline{N^{2}}$ & \% & $\underline{N^{2}}$ & \% & $\mathrm{N}^{2}$ & \% & $\underline{\text { N2}}$ & x & & \\
\hline cía & 45 & $0,5)$ & 118 & $3,6)$ & 24 & $10,9)$ & 41 & $(18,6)$ & 6 & $(2,7)$ & 31 & \\
\hline & 14 & & 32 & 56,2 & 2 & (is & 9 & $(15,5)$ & 3 & $(5,2)$ & 4 & \\
\hline & 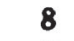 & $(12$, & 32 & 48,5 & 8 & $(12,1$ & 12 & $(18,2)$ & 2 & $(3,0)$ & 5 & \\
\hline & 2 & $(6,2)$ & 15 & $(46,9)$ & 1 & $(3,1)$ & 1 & $(3,1)$ & 4 & $(12,5)$ & 3 & \\
\hline & 5 & $(8,2)$ & 27 & $(44,3)$ & 4 & $(6,6)$ & 12 & $(19,7)$ & $i$ & $(1,6)$ & 7 & (i1, \\
\hline & 7 & $(25,0)$ & 14 & $(50,0)$ & 4 & $(14,3)$ & 9 & $(32,1)$ & 2 & $(7,1)$ & 4 & 14 \\
\hline cha & 8 & $(15,4)$ & 18 & $(34,6)$ & 3 & $(5,7)$ & 5 & $(9,6)$ & - & $\because$ & 3 & $(5$, \\
\hline & 20 & $(13$ & 68 & $(45,9)$ & 11 & $(7,4)$ & 20 & $(13,5)$ & 2 & $(1,3)$ & 16 & (10, \\
\hline & 95 & $(26$ & 227 & $(62,7)$ & 70 & $(19,3)$ & 100 & $(27,6)$ & 44 & $(12,1)$ & 66 & $(18$, \\
\hline & 4 & $(9$, & 17 & $(41,5)$ & 2 & (4 & 5 & $(12,2)$ & 2 & $(4,9)$ & 3 & (7), \\
\hline & 19 & $(18,1)$ & 46 & 43 & 8 & ) & 13 & $(12,4)$ & - & $\ldots$ & 8 & 7 \\
\hline & 3 & $(21,4)$ & 9 & (64, & 2 & & $\mathbf{3}$ & & - & - - & 3 & \\
\hline & 148 & 26 & 347 & 62 & 86 & & 197 & $(35,4)$ & 64 & $(11,5)$ & 145 & \\
\hline & 10 & 19 & 15 & 28 & 5 & $(9$ & 9 & $(17,3)$ & 1 & $(1,9)$ & 11 & \\
\hline & 7 & $26,9)$ & 13 & $(50,0)$ & 4 & (15, & 5 & $(19,2)$ & 4 & $(15,4)$ & 2 & 1, \\
\hline Pals & 35 & 28, & 66 & $(53,6)$ & 18 & (14 & 22 & $(17,9)$ & 12 & $(9,8)$ & 19 & (i5 \\
\hline Valencia & 19 & $(17,0)$ & 65 & $(58,0)$ & 13 & $(11,6)$ & 27 & $(24,1)$ & 5 & $(4,5)$ & 9 & \\
\hline Total & 449 & $21,8)$ & 1149 & $35,9)$ & 265 & 12,9) & 185 & $(23,6)$ & 152 & $(7,4)$ & 339 & 6, \\
\hline
\end{tabular}

C - Prétamo interbibliotecario

D - Reprosrafía de fondos propios

F - Reprografia de otros fondos nacionales o extranjeros

H - Búsquedas retrospectivas manuales

I - Búsquedas retrospectivas automatisadas

L - Cursos o seminarios

\section{Conclusiones}

Aunque en los distintos capítulos se han ido destacando los resultados parciales del análisis realizado, como conclusiones de conjunto se pueden deducir las siguientes:

1) Existe una gran desigualdad entre las diferentes comunidades autónomas con respecto a sus posibilidades de acceso directo a la información científica, tanto en relación al número de centros como al de recursos bibliográficos.

2) La mayor parte de las bibliotecas especializadas y centros de documentación de España son de reciente creación - menos de cincuenta años de antigüedad- y sólo un $5 \%$ tiene más de cien años.

3) La dependencia institucional de estos centros es mayoritariamente de organismos oficiales, y sólo un pequeño porcentaje está vinculado a entidades privadas.

4) A pesar del bajo número de centros que dependen de entidades privadas, sólo algo más del $40 \%$ del total del pais es de accesibilidad total.

5) Hay una marcada escasez de grandes bibliotecas, en cuanto al volumen de sus fondos tradicionales (libros y revistas), en particular en algunas comunidades autónomas, y una pobreza generalizada en cuanto a fondos destinados a búsqueda de información (revistas de resúmenes y obras de referencia). 
6) Como carencias más destacadas en los servicios que estos centros prestan, hay que mencionar que sólo algo más de un $20 \%$ realiza préstamo interbibliotecario y que casi un $45 \%$ no tiene establecido servicio de reproducción de sus propios fondos.

\section{Comentario final}

Cabe señalar que:

$\left.1^{\circ}\right)$ Los datos que han servido como punto de partida para el estudio muestran los recursos de información del pais en un momento dado y podrían haber cambiado algo; sin embargo, la política bibliotecaria del país no ha experimentado en el tiempo transcurrido desde la aparición del directorio un auge tal que haga presumible suponer un cambio acusado en el panorama de los centros de información científica del pais en ese periodo.

$\left.2^{\circ}\right)$ Como los datos proceden de cuestionarios contestados por los responsables de los distintos centros, pueden adolecer de alguna heterogeneidad, consecuencia del enfoque subjetivo de cada encuestado; no obstante, dado que la mayoria de los datos solicitados son concretos y en consecuencia necesitan poca interpretación, el subjetivismo parece poco probable, y

$3^{2}$ ) En todas las encuestas existe un número variable de sujetos que no colaboran, lo que se traduce en la obtención de unas cifras inferiores a las reales. En este caso, sin embargo, la autoridad del organismo solicitante de los datos incita a la contestación, por lo que se puede considerar válido el procentaje de respuestas.

Por todo ello, no parece aventurado suponer que la situación que el presente estudio pone de manifiesto es muy próxima a la que existe en la actualidad.

\section{Bibliografía}

1. Directorio de Centros de Documentación y Bibliotecas Especializadas. Ministerio de Educación y Ciencia. Madrid, 1987.

2. Nomenclatura Internacional de la Unesco para los campos de ciencia y tecnoloría. 24 verrión en español. UNESCO. Comisión Nacional de España. Madrid, 1987.

3. JIMENEZ, M. Las Bibliotecas Especializadas en España. Comunicación presentada en LIBER'89. 